\section{Generalized Hamilton's principle with fractional derivatives}

To cite this article: T M Atanackovi et al 2010 J. Phys. A: Math. Theor. 43255203

View the article online for updates and enhancements.

\section{Related content}

Variational problems with fractional derivatives: Euler-Lagrange equations

- Fractional variational principles with delay

Fractional variational calculus and the transversality conditions

\section{Recent citations}

\footnotetext{
- Perturbation to Noether symmetry for fractional dynamic systems of variable order

C. J. Song and Y. Zhang

Adiabatic Invariants for Generalized Fractional Birkhoffian Mechanics and Their Applications

C. J. Song

Conserved quantities and adiabatic invariants for fractional generalized Birkhoffian systems

Song Chuan-Jing and Zhang Yi
} 


\title{
Generalized Hamilton's principle with fractional derivatives
}

\author{
T M Atanacković ${ }^{1}$, S Konjik ${ }^{2}$, Lj Oparnica ${ }^{3}$ and S Pilipović ${ }^{2}$ \\ 1 Faculty of Technical Sciences, Institute of Mechanics, University of Novi Sad, \\ Trg Dositeja Obradovića 6, 21000 Novi Sad, Serbia \\ 2 Faculty of Sciences, Department of Mathematics and Informatics, University of Novi Sad, \\ Trg Dositeja Obradovića 4, 21000 Novi Sad, Serbia \\ 3 Institute of Mathematics, SANU, Kneza Mihaila 36, 11000 Belgrade, Serbia \\ E-mail: atanackovic@uns.ac.rs, sanja-konjik@uns.ac.rs, ljubicans@sbb.rs and \\ pilipovic@dmi.uns.ac.rs
}

Received 19 October 2009, in final form 18 April 2010

Published 27 May 2010

Online at stacks.iop.org/JPhysA/43/255203

\begin{abstract}
We generalize Hamilton's principle with fractional derivatives in the Lagrangian $L\left(t, y(t),{ }_{0} D_{t}^{\alpha} y(t), \alpha\right)$ so that the function $y$ and the order of the fractional derivative $\alpha$ are varied in the minimization procedure. We derive stationarity conditions and discuss them through several examples.
\end{abstract}

PACS numbers: $02.30, \mathrm{Xx}, 45.10, \mathrm{Hj}$

Mathematics Subject Classification: 49K05, 26A33

\section{Introduction}

Hamilton's principle is one of the basic principles of theoretical physics, where theory is often considered to be complete if its variational principle in the sense of Hamilton is known [4]. When Hamilton's principle is known, the information regarding the processes of a particular system is included into its Lagrangian. It could serve as a basis for obtaining first integrals via Nöther's theory, or to generate approximate solutions to the relevant system of equations by the use of the Ritz procedure ( $\operatorname{cf}[30]$ ).

In this paper we investigate necessary conditions for the existence of solutions of fractional variational problems (Euler-Lagrange equations) by the use of a generalized Hamilton's principle. Such investigations have been initiated in [25, 26], and continued in [1-5] and [8]; see also [24] for the importance of introducing fractional derivatives into the Lagrangian density of Hamilton's principle. In general, we refer to [9-16], [19, 22, 23] and [27-31] for different aspects of the calculus of variations and fractional calculus, motivations and applications.

When a fractional variational problem is studied, a natural question arises how can one choose $\alpha$, the order of fractional derivative, in order to achieve the minimal value of a 
functional under consideration. More generally, one can address the same question for any problem involving fractional operators. Usually, in application, a good choice of $\alpha$ is obtained by experiments or computational simulations. However, experimental results are theoretically not well confirmed. In this paper we propose a method which a priori gives values for $\alpha$ which optimize the considered variational problem following the fundamental minimization principle of Hamilton's action. In fact, we address the question of finding stationary points for Hamilton's action integral with fractional Lagrangian in a more general setting: we allow the stationarity of the action integral with respect to a set of admissible functions and with respect to the order of fractional derivatives, appearing in the Lagrangian. To our knowledge the problem when both $y$ and $\alpha$ are varied has not been analyzed so far. It leads to stationarity conditions as a basis for a generalized Hamilton's principle for the action integral

$I[y, \alpha]:=\int_{0}^{b} L\left(t, y(t),{ }_{0} D_{t}^{\alpha} y, \alpha\right) \mathrm{d} t, \quad y \in \mathcal{U}, \alpha \in A:=\left[0, \alpha_{0}\right], \quad \alpha_{0} \leqslant 1$,

where $\mathcal{U}$ is a set of admissible functions: find

$$
\min _{(y, \alpha) \in \mathcal{U} \times A} I[y, \alpha]
$$

or

$$
\min _{\alpha \in A}\left(\min _{y \in \mathcal{U}} I[y, \alpha]\right)
$$

or

$$
\min _{y \in \mathcal{U}}\left(\min _{\alpha \in A} I[y, \alpha]\right) .
$$

In this paper we analyze stationarity conditions for (2) and (3). Stationarity conditions with respect to $\alpha$ in (4) are more difficult, and, contrary to (3), this case is less natural in applications. Note that in (2), (3) and (4) one can look for maximums instead of minimums. So, our general problem is the determination of stationary points.

The paper is organized as follows. In the introduction we recall the definitions and properties of fractional derivatives. In section 2 we present a framework in which we shall study variational problems (2) and (3). Then in section 3 we derive stationarity conditions for (2). Section 4 is devoted to additional assumptions which provide equivalence of problems (2) and (3). Results which are obtained in the previous sections are illustrated by several examples in section 5. Moreover, examples of this section give further motivation for our investigation. In the last remark of section 5 we propose a new formulation of a fractional variational problem.

Throughout this paper we shall use the following notation. The mapping $(t, \alpha) \mapsto$ ${ }_{0} D_{t}^{\alpha}(y)$, which defines the left Riemann-Liouville fractional derivative of order $\alpha$, will be denoted by ${ }_{0} D_{t}^{\alpha} y$, or by ${ }_{0} D_{t}^{\alpha} y(t)$. Recall that

$$
{ }_{0} D_{t}^{\alpha} y:=\frac{1}{\Gamma(1-\alpha)} \frac{\mathrm{d}}{\mathrm{d} t} \int_{0}^{t} \frac{y(\tau)}{(t-\tau)^{\alpha}} \mathrm{d} \tau, \quad t \in[0, b], \quad 0 \leqslant \alpha<1,
$$

where $\Gamma$ is the Euler gamma function, and its existence is provided whenever

$$
[0, b] \ni t \mapsto \int_{0}^{t} \frac{y(\tau)}{(t-\tau)^{\alpha}} \mathrm{d} \tau
$$

is an absolutely continuous function. Recall that the space of absolutely continuous functions is denoted by $A C([0, b])$ and is supplied with the norm $\|f\|=\sup _{x \in[0, b]}|f(x)|$ (clearly, it is not a Banach space). For example, (5) is absolutely continuous if $y \in A C([0, b])$. However, there are some cases when with less regularity in $y$ we still have a well-defined operator of fractional differentiation (cf [27]). For instance, ${ }_{0} D_{t}^{\alpha} y$ exists for functions with integrable singularities 
(a continuous and locally integrable function $f$ in $(0, b]$ has an integrable singularity at $\tau=0$ of order $r<1$ if $\lim _{\tau \rightarrow 0} \tau^{r} f(\tau) \neq 0$ ). In particular, we can take $y(t)=t^{-\mu}, t \in(0, b]$ (for any $b>0$ ), $0<\mu<1$. Then we obtain the so-called Euler formula (cf [27, (2.26)])

$$
{ }_{0} D_{t}^{\alpha} t^{-\mu}=\frac{\Gamma(1-\mu)}{\Gamma(1-\mu-\alpha)} \frac{1}{t^{\mu+\alpha}}, \quad t \in(0, b] .
$$

The right Riemann-Liouville fractional derivative of order $\alpha$ is defined as

$$
{ }_{t} D_{b}^{\alpha} y:=\frac{1}{\Gamma(1-\alpha)}\left(-\frac{\mathrm{d}}{\mathrm{d} t}\right) \int_{t}^{b} \frac{y(\tau)}{(\tau-t)^{\alpha}} \mathrm{d} \tau, \quad t \in[0, b], \quad 0 \leqslant \alpha<1 .
$$

The conditions for its existence are similar as in the case of the left fractional derivative.

In the following we shall consider cases involving both fractional derivatives and work with integrable functions for which these derivatives (or one of them) exist. In such cases the notation ${ }_{0} D_{t}^{\alpha} y$, resp. ${ }_{t} D_{b}^{\alpha} y, t \in[0, b]$, means that $y$ and ${ }_{0} D_{t}^{\alpha} y$, resp. ${ }_{t} D_{b}^{\alpha} y$, are considered as integrable functions which can take values $+\infty$ or $-\infty$ at some points.

Recall [21] that ${ }_{0} D_{t}^{\alpha} y \rightarrow y^{\prime}$ and ${ }_{t} D_{b}^{\alpha} y \rightarrow-y^{\prime}$ in $\mathcal{C}([0, b])$, as $\alpha \rightarrow 1^{-}$, whenever $y \in \mathcal{C}^{1}([0, b])$.

Also, we shall make use of Caputo fractional derivatives. The left, resp. right, Caputo fractional derivative of order $\alpha \in[0,1)$ is defined as

${ }_{0}^{C} D_{t}^{\alpha} y:=\frac{1}{\Gamma(1-\alpha)} \int_{0}^{t} \frac{y^{\prime}(\tau)}{(t-\tau)^{\alpha}} \mathrm{d} \tau \quad$ resp. ${ }_{t}^{C} D_{b}^{\alpha} y:=\frac{1}{\Gamma(1-\alpha)} \int_{t}^{b} \frac{-y^{\prime}(\tau)}{(\tau-t)^{\alpha}} \mathrm{d} \tau$.

One can show that for $y \in A C([0, b])$ and $t \in[0, b]$,

${ }_{0} D_{t}^{\alpha} y={ }_{0}^{C} D_{t}^{\alpha} y+\frac{1}{\Gamma(1-\alpha)} \frac{y(0)}{(t-a)^{\alpha}}, \quad{ }_{t} D_{b}^{\alpha} y={ }_{t}^{C} D_{b}^{\alpha} y+\frac{1}{\Gamma(1-\alpha)} \frac{y(b)}{(b-t)^{\alpha}}$,

(cf e.g. [17]). Therefore, ${ }_{0} D_{t}^{\alpha} y={ }_{0}^{C} D_{t}^{\alpha} y$, resp. ${ }_{t} D_{b}^{\alpha} y={ }_{t}^{C} D_{b}^{\alpha} y$, whenever $y(0)=0$, resp. $y(b)=0$.

\section{Formulation of the problem}

We investigate stationary points of (1) for $\alpha \in\left[0, \alpha_{0}\right]$ and all admissible functions $y$, whose properties will be specified in the sequel. We shall distinguish two cases: $\alpha_{0}$ strictly less than 1 and $\alpha_{0}=1$. In the case $\alpha_{0}<1$, set

$$
\mathcal{U}_{l}:=\left\{\left.y \in L^{1}([0, b])\right|_{0} D_{t}^{\alpha} y \in L^{1}([0, b])\right\} .
$$

Obviously, $A C([0, b])$ is a subset of $\mathcal{U}_{l}$. In the case $\alpha_{0}=1$ we assume that $y \in \mathcal{U}_{l}$ and that, in addition, ${ }_{0} D_{t}^{1} y$ exists, and ${ }_{0} D_{t}^{1} y=y^{\prime}$ is an integrable function. Let us note that one can consider $\mathcal{U}_{l}$ defined with $L^{p}([0, b])$ (or their subspaces) instead of $L^{1}([0, b])$ (see remark 3.3).

In general, we shall use the notation

$$
\mathcal{U}:=\left\{y \in \mathcal{U}_{l} \mid y \text { satisfies specified boundary conditions }\right\} \text {. }
$$

We shall sometimes write $\mathcal{U}$ also for $\mathcal{U}_{l}$ (then the set of specified boundary conditions is empty).

In the following, the Lagrangian $L\left(t, y(t),{ }_{0} D_{t}^{\alpha} y, \alpha\right)$ (Lagrangian density, in physics) satisfies:

$$
\left.\begin{array}{c}
L \in \mathcal{C}^{1}([0, b] \times \mathbb{R} \times \mathbb{R} \times[0,1]) \\
\text { and } \\
t \mapsto \partial_{3} L\left(t, y(t),{ }_{0} D_{t}^{\alpha} y, \alpha\right) \in \mathcal{U}_{r}, \text { for every } y \in \mathcal{U}_{l}
\end{array}\right\}
$$

where $\mathcal{U}_{r}:=\left\{y \in L^{1}([0, b]) \mid{ }_{t} D_{b}^{\alpha} y \in L^{1}([0, b])\right\}$. 
Recall that our generalization of Hamilton's principle is realized through the determination of $\left(y^{*}, \alpha^{*}\right) \in \mathcal{U} \times A$ such that

$$
\min _{(y, \alpha) \in \mathcal{U} \times A} \int_{0}^{b} L\left(t, y(t),{ }_{0} D_{t}^{\alpha} y, \alpha\right) \mathrm{d} t=\int_{0}^{b} L\left(t, y^{*}(t),{ }_{0} D_{t}^{\alpha^{*}} y^{*}, \alpha^{*}\right) \mathrm{d} t .
$$

There are two special cases of (8). The first one is obtained when $A=\{1\}$. Then, since $\left.{ }_{0} D_{t}^{\alpha} y\right|_{\alpha=1}=y^{\prime}(t)$ for $y \in \mathcal{C}^{1}([0, b])$, the solution $y^{*}$ of (8) satisfies the classical Euler-Lagrange equation

$$
\frac{\mathrm{d}}{\mathrm{d} t} \frac{\partial L}{\partial y^{\prime}}-\frac{\partial L}{\partial y}=0
$$

If $A$ has a single element $A=\{\alpha\}, 0<\alpha<1$, then $\min _{(y, \alpha) \in \mathcal{U} \times\{\alpha\}} I[y, \alpha]$ leads to the fractional Euler-Lagrange equation $(\operatorname{cf}([1,5]))$

$$
{ }_{t} D_{b}^{\alpha} \frac{\partial L}{\partial_{0} D_{t}^{\alpha} y}+\frac{\partial L}{\partial y}=0
$$

We proceed with finding stationary points related to (1).

\section{Optimality conditions}

A necessary condition for the existence of solutions to the variational problem (8) is given in the following proposition.

Proposition 3.1. Let L satisfy (7). Then a necessary condition that functional (1) has an extremal point at $\left(y^{*}, \alpha^{*}\right) \in \mathcal{U} \times A$ is that $\left(y^{*}, \alpha^{*}\right)$ is a solution of the system of equations

$$
\begin{aligned}
& \frac{\partial L}{\partial y}+{ }_{t} D_{b}^{\alpha} \frac{\partial L}{\partial_{0} D_{t}^{\alpha} y}=0 \\
& \int_{0}^{b}\left(\frac{\partial L}{\partial_{0} D_{t}^{\alpha} y} G(y, \alpha)+\frac{\partial L}{\partial \alpha}\right) \mathrm{d} t=0,
\end{aligned}
$$

where

$G(y, \alpha)=\frac{\partial_{0} D_{t}^{\alpha} y}{\partial \alpha}=\frac{\mathrm{d}}{\mathrm{d} t}\left(f_{1} *_{t} y\right)(t, \alpha), f_{1}(t, \alpha)=\frac{1}{t^{\alpha} \Gamma(1-\alpha)}[\psi(1-\alpha)-\ln t], \quad t>0$, with the Euler function $\psi(z)=\frac{\mathrm{d}}{\mathrm{d} z} \ln \Gamma(z)$, and $\left(f_{1} *_{t} y\right)(t, \alpha)=\int_{0}^{t} f_{1}(\tau, \alpha) y(t-\tau) \mathrm{d} \tau$.

Proof. Let $\left(y^{*}, \alpha^{*}\right)$ be an element of $\mathcal{U} \times A$ for which $I[y, \alpha]$ has an extremal value. Let $y(t)=y^{*}(t)+\varepsilon_{1} f(t), \alpha=\alpha^{*}+\varepsilon_{2}, \varepsilon_{1}, \varepsilon_{2} \in \mathbb{R}$, with $f \in \mathcal{U}_{l}$, and the boundary conditions on $f$ are specified so that the varied path $y^{*}+\varepsilon_{1} f$ is an element of $\mathcal{U}$. Then $I[y, \alpha]=I\left[y^{*}+\varepsilon_{1} f, \alpha^{*}+\varepsilon_{2}\right]=: I\left(\varepsilon_{1}, \varepsilon_{2}\right)$. A necessary condition for an extremal value of $I[y, \alpha]$ is

Therefore we obtain

$$
\left.\frac{\partial I\left(\varepsilon_{1}, \varepsilon_{2}\right)}{\partial \varepsilon_{1}}\right|_{\varepsilon_{1}=0, \varepsilon_{2}=0}=0,\left.\quad \frac{\partial I\left(\varepsilon_{1}, \varepsilon_{2}\right)}{\partial \varepsilon_{2}}\right|_{\varepsilon_{1}=0, \varepsilon_{2}=0}=0
$$

$$
\begin{aligned}
& \int_{a}^{b}\left(\frac{\partial L}{\partial y} f(t)+\frac{\partial L}{\partial_{0} D_{t}^{\alpha} y}{ }_{0} D_{t}^{\alpha} f\right) \mathrm{d} t=0, \\
& \int_{0}^{b}\left(\frac{\partial L}{\partial_{0} D_{t}^{\alpha} y} \frac{\partial_{0} D_{t}^{\alpha} y}{\partial \alpha}+\frac{\partial L}{\partial \alpha}\right) \mathrm{d} t=0 .
\end{aligned}
$$


Applying the fractional integration by parts formula (cf [17]):

$$
\int_{0}^{b} g(t) \cdot{ }_{0} D_{t}^{\alpha} f(t) \mathrm{d} t=\int_{0}^{b} f(t) \cdot{ }_{t} D_{b}^{\alpha} g(t) \mathrm{d} t
$$

to (11), (11) is transformed into

$$
\int_{0}^{b}\left(\frac{\partial L}{\partial y}+{ }_{t} D_{b}^{\alpha} \frac{\partial L}{\partial_{0} D_{t}^{\alpha} y}\right) f(t) \mathrm{d} t=0
$$

From this equation, using the fundamental lemma of the calculus of variations (see [9, p 115]), we conclude that condition (9) holds for the optimal values $y^{*}$ and $\alpha^{*}$. In (10) the term $\frac{\partial_{0} D_{t}^{\alpha} y}{\partial \alpha}$ is transformed by the use of the expression

$$
\begin{aligned}
\frac{\partial_{0} D_{t}^{\alpha} y}{\partial \alpha} & =\psi(1-\alpha)_{0} D_{t}^{\alpha} y-\frac{1}{\Gamma(1-\alpha)} \frac{\mathrm{d}}{\mathrm{d} t} \int_{0}^{t} \frac{\ln (t-\tau) y(\tau)}{(t-\tau)^{\alpha}} \mathrm{d} \tau \\
& =\frac{\mathrm{d}}{\mathrm{d} t}\left(f_{1} *_{t} y\right)(t, \alpha) \\
& =G(y, \alpha), \quad(y, \alpha) \in \mathcal{U} \times A
\end{aligned}
$$

(cf [6, p 592]). We obtain (10) by substituting (14) into (12).

Remark 3.2. In general, while solving equations (9) and (10), the most delicate task is the calculation of the expression $\frac{\partial_{0} D_{t}^{\alpha} y}{\partial \alpha}$. Although its general form (14) has been derived in [6, p 592], various difficulties can still appear. We illustrate this by examples in section 5 . However, the simplified form of $\frac{\partial_{0} D_{t}^{\alpha} y}{\partial \alpha}$, in some special cases, is important.

Already in [6] it has been shown that for $y \in A C([0, b])$

$$
\begin{aligned}
\left.\frac{\partial_{0} D_{t}^{\alpha} y}{\partial \alpha}\right|_{\alpha=0^{+}} & =-(\gamma+\ln t) y(0)-\int_{0}^{t}(\gamma+\ln \tau) y(t-\tau) \mathrm{d} \tau \\
& =-(\gamma+\ln t) y(t)+\int_{0}^{t} \frac{y(t)-y(t-\tau)}{\tau} \mathrm{d} \tau,
\end{aligned}
$$

where $\gamma=0.5772156 \ldots$ is the Euler constant. (Another form of $\left.\frac{\partial_{0} D_{t}^{\alpha} y}{\partial \alpha}\right|_{\alpha=0^{+}}$is also given in [31, p 111].)

Let us obtain a simplified form of $\frac{\partial_{0} D_{t}^{\alpha} y}{\partial \alpha}$ at $\alpha=1^{-}$. In order to do that we use the method proposed in [28]. We recall the expansion of $(t-\tau)^{\varepsilon} / \Gamma(1+\varepsilon)$ with respect to $\varepsilon$, at $\varepsilon=0$, with $\tau<t$ (cf [28, p 401]), which will be used in the following:

$$
\frac{(t-\tau)^{\varepsilon}}{\Gamma(1+\varepsilon)}=\frac{\mathrm{e}^{\varepsilon \ln (t-\tau)}}{\Gamma(1+\varepsilon)}=1+\varepsilon(\gamma+\ln (t-\tau))+o(\varepsilon) .
$$

Now assume that $y \in C^{2}([0, b])$. Then, as in [23], for $t \in[0, b]$,

$$
\begin{aligned}
{ }_{0} D_{t}^{\alpha} y & =\frac{1}{\Gamma(1-\alpha)} \frac{\mathrm{d}}{\mathrm{d} t} \int_{0}^{t} \frac{y(\tau)}{(t-\tau)^{\alpha}} \mathrm{d} \tau \\
& =\frac{y(0)}{\Gamma(1-\alpha) t^{\alpha}}+\frac{1}{\Gamma(1-\alpha)} \int_{0}^{t} \frac{y^{(1)}(\tau)}{(t-\tau)^{\alpha}} \mathrm{d} \tau \\
& =\frac{y(0)}{\Gamma(1-\alpha) t^{\alpha}}+\frac{y^{(1)}(0)}{\Gamma(2-\alpha) t^{\alpha-1}}+\frac{1}{\Gamma(2-\alpha)} \int_{0}^{t}(t-\tau)^{1-\alpha} y^{(2)}(\tau) \mathrm{d} \tau
\end{aligned}
$$

With $\alpha=1-\varepsilon$ we obtain

$$
{ }_{0} D_{t}^{1-\varepsilon} y=\frac{y(0)}{\Gamma(\varepsilon) t^{1-\varepsilon}}+\frac{y^{(1)}(0) t^{\varepsilon}}{\Gamma(1+\varepsilon)}+\frac{1}{\Gamma(1+\varepsilon)} \int_{0}^{t}(t-\tau)^{\varepsilon} y^{(2)}(\tau) \mathrm{d} \tau \text {. }
$$


From (17) and (16) it follows that

$$
\begin{aligned}
\left.\frac{\partial_{0} D_{t}^{\alpha} y}{\partial \alpha}\right|_{\alpha=1^{-}} & =-\left.\frac{\partial_{0} D_{t}^{1-\varepsilon} y}{\partial \varepsilon}\right|_{\varepsilon=0^{+}} \\
& =-\frac{y(0)}{t}-y^{(1)}(0) \ln t-\gamma y^{(1)}(t)-\int_{0}^{t} y^{(2)}(\tau) \ln (t-\tau) \mathrm{d} \tau .
\end{aligned}
$$

Assuming $y(0)=0$ in (18), we recover the results presented in [28] and [29] for the Caputo fractional derivative. Since ${ }_{0} D_{t}^{\alpha} y={ }_{0}^{C} D_{t}^{\alpha} y$ when $y(0)=0$, it follows that with $y(0)=0(18)$ becomes

$$
\left.\frac{\partial_{0} D_{t}^{\alpha} y}{\partial \alpha}\right|_{\alpha=1^{-}}=\left.\frac{\partial_{0}^{C} D_{t}^{\alpha} y}{\partial \alpha}\right|_{\alpha=1^{-}}=-y^{(1)}(0) \ln t-\gamma y^{(1)}(t)-\int_{0}^{t} y^{(2)}(\tau) \ln (t-\tau) \mathrm{d} \tau .
$$

Remark 3.3. Functional (1) is a special case of the class of functionals with Lagrangians depending on linear operators, see [12, p 51]. Indeed, suppose that the Lagrangian $L$ depends on $t, y$ and $\mathcal{L} y$, where $\mathcal{L}: M \rightarrow L^{p}([0, b]), p \in[1,+\infty)$, is a linear operator acting on a set of admissible functions $M$, which is linear, open and dense in $L^{p}([0, b])$ (i.e. $\mathcal{L}$ belongs to $\operatorname{Lin}\left(M, L^{p}([0, b])\right)$, the space of continuous, linear functions with the uniform norm). Suppose that $L$ is continuously differentiable with respect to $t$ and $y$ and twice continuously differentiable with respect to $\mathcal{L} y$. Moreover, assume that the function $t \mapsto L(t, y(t), \mathcal{L} y(t))$, $t \in[0, b]$, is continuous, for all $y \in M$. Then the Euler-Lagrange equation reads

$$
\frac{\partial L}{\partial y}+\mathcal{L}^{*} \frac{\partial L}{\partial(\mathcal{L} y)}=0
$$

where $\mathcal{L}^{*}$ denotes the adjoint operator of $\mathcal{L}$. In the case when $\mathcal{L}$ is the left Riemann-Liouville operator ${ }_{0} D_{t}^{\alpha}$, with the adjoint ${ }_{t} D_{b}^{\alpha}$, the latter equation coincides with (9).

If instead of $\mathcal{L}$ one considers a family $\left\{\mathcal{L}_{\alpha}, \alpha \in A\right\}$, where $A=[0,1]$ (or some other interval), and the mapping $A \rightarrow \operatorname{Lin}\left(M, L^{p}([0, b])\right), \alpha \mapsto \mathcal{L}_{\alpha}$, is differentiable, then a more general problem of finding stationary points with respect to $y$ and $\alpha$ can be formulated. In that case, one can derive the second stationarity condition similar to (10):

$$
\int_{0}^{b}\left(\frac{\partial L}{\partial \mathcal{L}} \frac{\partial \mathcal{L}}{\partial \alpha}+\frac{\partial L}{\partial \alpha}\right) \mathrm{d} t=0
$$

\section{Equivalent problems}

In this section we shall give conditions which show that problems (2) and (3) coincide.

Proposition 4.1. Let the Lagrangian L satisfy (7). Assume that for every $\alpha \in[0,1]$ there is a unique $y^{*}(t, \alpha) \in \mathcal{U}$, a solution to (9), and that the mapping $\alpha \mapsto y^{*}(t, \alpha)$ is differentiable as a mapping from $[0,1]$ to $\mathcal{U}$. Then the problem $\min _{(y, \alpha) \in \mathcal{U} \times A} I[y, \alpha]$ is equivalent to the problem $\min _{\alpha \in A}\left(\min _{y \in \mathcal{U}} I[y, \alpha]\right)$.

Proof. As we have shown in proposition 3.1, any solution to the problem $\min _{(y, \alpha) \in \mathcal{U} \times A} I[y, \alpha]$ satisfies the system (9)-(10). It can be solved as follows. We first solve (9) and the corresponding boundary conditions to obtain $y^{*}=y^{*}(t, \alpha)$. According to the assumption, the solution $y^{*}$ is unique. Then we insert $y^{*}$ in (10) to obtain $\alpha^{*}$. In this case, the functional 
$I[y, \alpha]$ becomes a functional depending only on $\alpha, \alpha \mapsto I\left[y^{*}(t, \alpha), \alpha\right]=I[\alpha]$, and therefore (10) transforms into the total derivative of $I[\alpha]$ since

$$
\begin{aligned}
\frac{\mathrm{d} I[\alpha]}{\mathrm{d} \alpha} & =\left.\frac{\mathrm{d} I[\alpha+\varepsilon]}{\mathrm{d} \varepsilon}\right|_{\varepsilon=0} \\
& =\int_{0}^{1}\left[\frac{\partial L}{\partial y} \frac{\partial y}{\partial \alpha}+\frac{\partial L}{\partial_{0} D_{t}^{\alpha} y}\left({ }_{0} D_{t}^{\alpha}\left(\frac{\partial y}{\partial \alpha}\right)+\left(\frac{\partial}{\partial \alpha}{ }_{0} D_{t}^{\alpha}\right) y\right)+\frac{\partial L}{\partial \alpha}\right] \mathrm{d} t \\
& =\int_{0}^{1}\left[\frac{\partial y}{\partial \alpha}\left(\frac{\partial L}{\partial y}+{ }_{t} D_{b}^{\alpha} \frac{\partial L}{\partial_{0} D_{t}^{\alpha} y}\right)+\frac{\partial L}{\partial_{0} D_{t}^{\alpha} y}\left(\frac{\partial}{\partial \alpha}{ }_{0} D_{t}^{\alpha}\right) y+\frac{\partial L}{\partial \alpha}\right] \mathrm{d} t \\
& =\int_{0}^{1}\left(\frac{\partial L}{\partial_{0} D_{t}^{\alpha} y}\left(\frac{\partial}{\partial \alpha}{ }_{0} D_{t}^{\alpha}\right) y+\frac{\partial L}{\partial \alpha}\right) \mathrm{d} t
\end{aligned}
$$

where we used fractional integration by parts formula (13) in the third, and equation (9) in the last equality. This proves the claim.

The following simple assertion is of particular interest.

Proposition 4.2. Let L satisfy (7). Assume that for every $\alpha \in[0,1]$ there exists a unique $y_{\alpha} \in \mathcal{U}$, a solution to the fractional variational problem (8), and that $I\left[y_{\alpha}, \alpha\right]$ is the corresponding minimal value of the functional I. Assume additionally that

$$
\left.\frac{\mathrm{d} I}{\mathrm{~d} \alpha}(y, \alpha)\right|_{y=y_{\alpha}}>0, \quad \forall y_{\alpha} \in \mathcal{U}
$$

Then the minimal and maximal value of the functional $I[y, \alpha]$ is attained at $\alpha=0$ resp. at $\alpha=1$.

Proof. Under the above assumptions we have that

$$
I\left[y_{0}, 0\right] \leqslant I\left[y_{\alpha}, 0\right] \leqslant I\left[y_{\alpha}, \alpha\right] \leqslant I\left[y_{1}, \alpha\right] \leqslant I\left[y_{1}, 1\right], \quad \forall \alpha \in[0,1],
$$

which proves the claim.

Remark 4.3. The same argument can be applied to the case when $\mathrm{d} I / \mathrm{d} \alpha<0$, for any fixed $y_{\alpha} \in \mathcal{U}$, i.e. when $I$ is a decreasing function of $\alpha$, for any fixed $y_{\alpha} \in \mathcal{U}$. In that case the minimal and maximal value of $I$ is at $\alpha=1$, resp. $\alpha=0$.

\section{Examples}

\subsection{Examples with Lagrangians linear in $y$}

Example 5.1. Consider the action integral for the inertial motion (no force acting) of a material point of the form

$$
I[y, \alpha]:=\int_{0}^{1}\left({ }_{0} D_{t}^{\alpha} y\right)^{2} \mathrm{~d} t, \quad(y, \alpha) \in \mathcal{U} \times A,
$$

where $\mathcal{U}:=\left\{y \in \mathcal{U}_{l} \mid y(0)=0, y(1)=1\right\}$ and $A=[0,1]$.

Obviously, the minimal value of $I[y, \alpha]$ is zero, and it is attained whenever ${ }_{0} D_{t}^{\alpha} y=0$. Solutions to the equation ${ }_{0} D_{t}^{\alpha} y=0$ are of the form $y(t)=C \cdot t^{1-\alpha}, t \in[0,1], C \in \mathbb{R}$ (cf [27]). All solutions satisfy the Euler-Lagrange equation

$$
{ }_{t} D_{1}^{\alpha}\left({ }_{0} D_{t}^{\alpha} y\right)=0 \text {. }
$$


The stationarity condition (10) reads

and is automatically satisfied.

$$
\int_{0}^{1}{ }_{0} D_{t}^{\alpha} y\left(\psi(1-\alpha)_{0} D_{t}^{\alpha} y-\frac{1}{\Gamma(1-\alpha)} \frac{\mathrm{d}}{\mathrm{d} t} \int_{0}^{t} \frac{\ln (t-\tau) y(\tau)}{(t-\tau)^{\alpha}} \mathrm{d} \tau\right) \mathrm{d} t=0
$$

Note that $C \cdot t^{1-\alpha} \in \mathcal{U}_{l}$, for all $C \in \mathbb{R}$, but only $t^{1-\alpha} \in \mathcal{U}$. Hence, we conclude that $\left(y^{*}, \alpha^{*}\right)=\left(t^{1-\alpha}, \alpha\right), \alpha \in[0,1]$, are solutions to the variational problem $I[y, \alpha] \rightarrow$ min, for $I$ defined by (20).

Remark 5.2. If $L\left(t, y(t),{ }_{0} D_{t}^{\alpha} y, \alpha\right)=\left({ }_{0} D_{t}^{\alpha} y\right)^{2}+\left(\alpha-\alpha_{0}\right)^{2}$, for a fixed $\alpha_{0} \in(0,1)$, then the problem $\int_{0}^{1} L\left(t, y(t),{ }_{0} D_{t}^{\alpha} y, \alpha\right) \mathrm{d} t \rightarrow \min$ has a unique minimizer $\left(y^{*}, \alpha^{*}\right)=\left(t^{1-\alpha_{0}}, \alpha_{0}\right)$.

Example 5.3. Let the Lagrangian $L$ be of the form

$$
L\left(t, y(t),{ }_{0} D_{t}^{\alpha} y, \alpha\right):=\left({ }_{0} D_{t}^{\alpha} y\right)^{2}-c \cdot y, \quad c \in \mathbb{R}
$$

and let $\mathcal{U}=\left\{y \in \mathcal{U}_{l} \mid y(0)=0\right\}, A=[0,1]$, for the variational problem

$$
\min _{(y, \alpha) \in \mathcal{U} \times A} I[y, \alpha]=\min _{(y, \alpha) \in \mathcal{U} \times A} \int_{0}^{1}\left(\left({ }_{0} D_{t}^{\alpha} y\right)^{2}-c \cdot y(t)\right) \mathrm{d} t .
$$

Equations (9) and (10) become

$$
{ }_{t} D_{10}^{\alpha} D_{t}^{\alpha} y=c, \quad \int_{0}^{1}{ }_{0} D_{t}^{\alpha} y \cdot \frac{\partial_{0} D_{t}^{\alpha} y}{\partial \alpha} \mathrm{d} t=0 .
$$

Equation (21) could be solved as follows. First, one introduces a substitution $z(t)={ }_{0} D_{t}^{\alpha} y$, $t \in[0,1]$, and solve ${ }_{t} D_{1}^{\alpha} z=c$ :

$$
z(t)=c \cdot \frac{(1-t)^{\alpha}}{\Gamma(1-\alpha)}, \quad t \in[0,1], \quad \alpha \in A
$$

Therefore,

$$
{ }_{0} D_{t}^{\alpha} y(t)=c \cdot \frac{(1-t)^{\alpha}}{\Gamma(1-\alpha)}, \quad t \in[0,1], \quad \alpha \in A
$$

Recall that

$$
{ }_{0} I_{t}^{\alpha} y:=\frac{1}{\Gamma(\alpha)} \int_{0}^{t}(t-\tau)^{\alpha} y(\tau) \mathrm{d} \tau, \quad t \in[0,1], \quad \alpha \in A,
$$

and apply it on the both sides of (22). Using [27, theorem 2.4], i.e. ${ }_{0} I_{t}^{\alpha}\left({ }_{0} D_{t}^{\alpha} y\right)=$ $y(t)-\left.\frac{t^{\alpha-1}}{\Gamma(\alpha)}{ }_{0} I_{t}^{1-\alpha} y\right|_{t=0}$, one obtains

$$
\begin{aligned}
y(t, \alpha) & =\frac{c}{\Gamma(\alpha) \Gamma(1+\alpha)} \int_{0}^{t}(t-\tau)^{\alpha-1}(1-\tau)^{\alpha} \mathrm{d} \tau \\
& =\frac{c}{\Gamma(1+\alpha)} \sum_{p=0}^{\infty} \frac{\Gamma(p-\alpha) \Gamma(1+p)}{\Gamma(-\alpha) p ! \Gamma(1+p+\alpha)} t^{p+\alpha}, \quad t \in[0,1], \quad \alpha \in A .
\end{aligned}
$$

This solution is unique and belongs to $\mathcal{U}$. Since $\alpha \mapsto y(t, \alpha)$ is differentiable, proposition 4.1 holds.

We substitute the obtained $y(t, \alpha)$ into $I[y, \alpha]$ which yields

$$
\begin{aligned}
I[\alpha] & =\int_{0}^{1}\left(\left(c \cdot \frac{(1-t)^{\alpha}}{\Gamma(1-\alpha)}\right)^{2}-\frac{c^{2}}{\Gamma(\alpha) \Gamma(1+\alpha)} \int_{0}^{t}(t-\tau)^{\alpha-1}(1-\tau)^{\alpha} \mathrm{d} \tau\right) \mathrm{d} t \\
& \left.=\int_{0}^{1}\left(\left(c \cdot \frac{(1-t)^{\alpha}}{\Gamma(1-\alpha)}\right)\right)^{2}-\frac{c^{2}}{\Gamma(1+\alpha)} \sum_{p=0}^{\infty} \frac{\Gamma(p-\alpha) \Gamma(1+p)}{\Gamma(-\alpha) p ! \Gamma(1+p+\alpha)} t^{p+\alpha}\right) \mathrm{d} t .
\end{aligned}
$$


Simple numerical calculations show that $I[\alpha]$ is an increasing function and attains extremal values at the boundaries.

Remark 5.4. Equation (21) represents a fractional generalization of the equation of motion for a material point (with unit mass) under the action of constant force equal to $c$. Our result shows that an optimal value of Hamilton's action is attained for $\alpha=1$, that is for integer order dynamics. We note that different generalizations of the classical equation of motion can be found in [18], where the problem ${ }_{0} D_{t}^{\alpha} y=c, 1<\alpha \leqslant 2$, was analyzed.

\subsection{Examples with Lagrangians linear in ${ }_{0} D_{t}^{\alpha} y$}

Example 5.5. Let

$$
L\left(t, y(t),{ }_{0} D_{t}^{\alpha} y, \alpha\right):=\Gamma(1-\alpha)_{0} D_{t}^{\alpha} y-\frac{1}{2} c y^{2}, \quad c>0, c \neq 1,
$$

and consider the problem of finding stationary points for functional (1), where $\mathcal{U}:=\{y \in$ $\left.\mathcal{U}_{l} \mid y(0)=\frac{1}{c}\right\}$ and $\alpha_{0}<\frac{1}{2}$. Note that $L$ satisfies the so-called primary constraint in Dirac's classification of systems with constraints (cf [15]). In the setting of fractional derivatives such Lagrangians have been recently treated in [7] and [20].

Equations (9) and (10) become

$$
\Gamma(1-\alpha)_{t} D_{1}^{\alpha} 1-c y=0
$$

and

$$
\int_{0}^{1}\left(\Gamma(1-\alpha) \frac{\partial_{0} D_{t}^{\alpha} y}{\partial \alpha}+\frac{\partial \Gamma(1-\alpha)}{\partial \alpha}\right) \mathrm{d} t=0 .
$$

Equation (24) has a unique solution $y^{*}=\frac{1}{c(1-t)^{\alpha}}, t \in[0,1], \alpha \in\left[0, \alpha_{0}\right]$. This implies

$$
\begin{aligned}
I\left[y^{*}, \alpha\right] & =\int_{0}^{1}\left[\frac{\mathrm{d}}{\mathrm{d} t} \int_{0}^{t} \frac{\mathrm{d} \tau}{c(1-\tau)^{\alpha}(t-\tau)^{\alpha}}-\frac{1}{2 c(1-t)^{2 \alpha}}\right] \mathrm{d} t \\
& =\left.\int_{0}^{t} \frac{\mathrm{d} \tau}{c(1-\tau)^{\alpha}(t-\tau)^{\alpha}}\right|_{0} ^{1}-\int_{0}^{1} \frac{1}{2 c(1-t)^{2 \alpha}} \mathrm{d} t \\
& =\int_{0}^{1} \frac{\mathrm{d} \tau}{c(1-\tau)^{2 \alpha}}-\int_{0}^{1} \frac{1}{2 c(1-t)^{2 \alpha}} \mathrm{d} t \\
& =\frac{1}{2 c} \int_{0}^{1} \frac{1}{(1-t)^{2 \alpha}} \mathrm{d} t .
\end{aligned}
$$

Since $\alpha_{0}<1 / 2$ we have that $I\left[y^{*}, \alpha\right]$ exists and is an increasing function with respect to $\alpha$. Hence, $I\left[y^{*}, \alpha\right]$ attains its minimal value at $\alpha=0$, and it equals $1 /(2 c)$. We also have that the maximal value of $I\left[y^{*}, \alpha\right]$ is attained at $\alpha_{0}$.

Example 5.6. Let $\mathcal{U}:=\left\{y \in \mathcal{U}_{l} \mid y(0)=0\right\}, c \neq 0$ and let $L$ be of the form

$$
L\left(t, y(t),{ }_{0} D_{t}^{\alpha} y, \alpha\right):=c \cdot{ }_{0} D_{t}^{\alpha} y+f(y(t)), \quad t \in[0,1],
$$

where the properties of $f$ are going to be specified.

In this example we are dealing with integrable functions which can take values $+\infty$ or $-\infty$ at some points. We are going to analyze stationary points of

$$
I[y, \alpha]=\int_{0}^{1}\left(c \cdot{ }_{0} D_{t}^{\alpha} y(t)+f(y(t))\right) \mathrm{d} t .
$$


Equations (9) and (10) become

$$
\begin{aligned}
& { }_{t} D_{1}^{\alpha} c+\frac{\partial f}{\partial y}=0 \\
& c \cdot \int_{0}^{1} \frac{\partial_{0} D_{t}^{\alpha} y}{\partial \alpha} \mathrm{d} t=0 .
\end{aligned}
$$

Since ${ }_{t} D_{1}^{\alpha} c=\frac{c}{\Gamma(1-\alpha)(1-t)^{\alpha}}, t \in[0,1]$, we see that in order to solve (27)-(28) we have to assume that $f \in \mathcal{C}^{1}(\mathbb{R})$, and that $f^{\prime}$ is invertible so that $t \mapsto\left(f^{\prime}\right)^{-1}\left(\frac{c}{\Gamma(1-\alpha)(1-t)^{\alpha}}\right) \in \mathcal{U}_{l}$. Then equation (27) is solvable with respect to $y$ :

$$
y_{c}(t, \alpha)=\left(\frac{\partial f}{\partial y}\right)^{-1}\left(\frac{c}{\Gamma(1-\alpha)(1-t)^{\alpha}}\right), \quad t \in[0,1] .
$$

Since $c \neq 0$, (28) implies $\int_{0}^{1} \frac{\partial_{0} D_{t}^{\alpha} y}{\partial \alpha} \mathrm{d} t=0$. Thus,

$$
\begin{aligned}
0 & =\int_{0}^{1} \frac{\partial_{0} D_{t}^{\alpha} y}{\partial \alpha} \mathrm{d} t=\int_{0}^{1} G(y, \alpha)(t) \mathrm{d} t=\int_{0}^{1} \frac{\mathrm{d}}{\mathrm{d} t}\left(f_{1} *_{t} y\right)(t, \alpha) \mathrm{d} t \\
& =\left.f_{1} *_{t} y(t, \alpha)\right|_{t=1}-\left.f_{1} *_{t} y(t, \alpha)\right|_{t=0}=\left.f_{1} *_{t} y(t, \alpha)\right|_{t=1},
\end{aligned}
$$

where we have used that $f_{1} \in L^{1}([0,1])$ and that $y \in \mathcal{U}$. Substitution of (29) into (30) gives $\left.\left(f_{1} * y_{c}(t, \alpha)\right)(t)\right|_{t=1}=0$ or

$$
\int_{0}^{1} \frac{\psi(1-\alpha)-\ln (1-\tau)}{\Gamma(1-\alpha)(1-\tau)^{\alpha}}\left(\frac{\partial f}{\partial y}\right)^{-1}\left(\frac{c}{\Gamma(1-\alpha)(\tau-1)^{\alpha}}\right) \mathrm{d} \tau=0
$$

Solving this equation is obviously difficult. Hence, we consider some special cases.

(a) $f(y(t)):=d \cdot \frac{y(t)^{2}}{2}, t \in[0,1], \mathrm{d} \in \mathbb{R}$. Then the Lagrangian is

$$
L\left(t, y(t),{ }_{0} D_{t}^{\alpha} y, \alpha\right)=c \cdot{ }_{0} D_{t}^{\alpha} y(t)+d \cdot \frac{y(t)^{2}}{2},
$$

and

$$
y_{c}(t, \alpha)=-\frac{1}{d} \frac{c}{\Gamma(1-\alpha)(1-t)^{\alpha}}, \quad t \in[0,1] .
$$

Also, (31) becomes

$$
\int_{0}^{1} \frac{\psi(1-\alpha)-\ln (1-\tau)}{\Gamma(1-\alpha)^{2}(1-\tau)^{2 \alpha}} \mathrm{d} \tau=0
$$

By a simple numerical calculation one shows that this equation does not have any solution for $\alpha \in(0,1)$. Hence, in this case there does not exist any point $(y, \alpha)$ which is an extremal of functional $I[y, \alpha]$.

(b) $f(y(t)):=\ln y(t), t \in[0,1]$. Then (27) becomes

$$
\frac{c}{\Gamma(1-\alpha)(1-t)^{\alpha}}=\frac{1}{y}
$$

and therefore

$$
y=\frac{\Gamma(1-\alpha)}{c}(1-t)^{\alpha} \in A C([0,1]) .
$$

In this particular case we take the set of admissible functions to be $\mathcal{U}:=\left\{y \in \mathcal{U}_{l} \mid y(1)=0\right\}$. Using (30), equation (28) reads

$$
\int_{0}^{1}(\psi(1-\alpha)-\ln (1-\tau)) \mathrm{d} \tau=0
$$

10 
which, after integration, yields $\psi(\alpha-1)=1$. A unique solution of this equation in $(0,1)$ is $\alpha=0.604 \ldots$.

Therefore, a unique stationary point of

$$
I[y, \alpha]=\int_{0}^{1}\left(c \cdot{ }_{0} D_{t}^{\alpha} y+\ln y(t)\right) \mathrm{d} t
$$

is the point $(y, \alpha)=\left(\frac{\Gamma(0,396)}{c}(1-t)^{\alpha} ; 0,604\right)$.

Remark 5.7. So far, we have considered variational problems defined via functionals of type (1). In fact, we have allowed fractional derivatives of functions to appear in Lagrangians. The natural generalization of such problems consists of replacing the Lebesgue integral in (1) by the Riemann-Liouville fractional integral. More precisely, for $\beta>0$ set

$$
\begin{aligned}
I_{\beta}[y, \alpha] & :={ }_{0} I_{b}^{\beta} L\left(t, y(t),{ }_{0} D_{t}^{\alpha} y, \alpha\right) \\
& =\frac{1}{\Gamma(\beta)} \int_{0}^{b}(b-t)^{\beta-1} L\left(t, y(t),{ }_{0} D_{t}^{\alpha} y, \alpha\right) \mathrm{d} t, \quad t \in(0, b) .
\end{aligned}
$$

Then the fractional variational problem consists of finding extremal values of the functional $I_{\beta}[y, \alpha]$.

In the above construction we have used the left Riemann-Liouville fractional integral of order $\beta$ (which, in general, differs from the order of fractional differentiation $\alpha$ ), evaluated at $t=b$. The choice $\beta=1$ turns us back to the problem (1).

The study of such fractional variational problems is reduced to the case we have already considered in the following way. It suffices to redefine the Lagrangian as

$$
L_{1}\left(t, y(t),{ }_{0} D_{t}^{\alpha} y, \alpha, \beta\right):=\frac{1}{\Gamma(\beta)}(b-t)^{\beta-1} L\left(t, y(t),{ }_{0} D_{t}^{\alpha} y, \alpha\right) .
$$

Then we have to consider the functional

$$
I_{\beta}[y, \alpha]=\int_{0}^{b} L_{1}\left(t, y(t),{ }_{0} D_{t}^{\alpha} y, \alpha, \beta\right) \mathrm{d} t .
$$

In case $\beta>1, L_{1}$ is of the same regularity as $L$, so the straightforward application of the results derived in the previous sections to the Lagrangian $L_{1}$ leads to the optimality conditions for the variational problem defined via functional (32). However, when $0<\beta<1$, continuity as well as differentiability of $L_{1}$ with respect to $t$ may be violated (which depends of the explicit form of $L$ ), and hence it may not be possible to use the theory developed so far.

\section{Conclusion}

We formulated Hamilton's principle so that the order of the derivative in the Lagrangian is also subject to variation. The stationarity conditions are derived in (9) and (10). We introduced additional assumptions which resulted in equivalent problems, simpler for solving. Several examples are given in order to illustrate the theory presented in the paper. We concluded our work with a consideration of Hamilton's principle defined in terms of Riemann-Liouville fractional integrals.

\section{Acknowledgments}

This work is supported by Projects 144016 and 144019 of the Serbian Ministry of Science and START-project Y-237 of the Austrian Science Fund. 


\section{References}

[1] Agrawal O P 2002 Formulation of Euler-Lagrange equations for fractional variational problems J. Math. Anal. Appl. 272 368-79

[2] Agrawal O P 2006 Fractional variational calculus and the transversality conditions J. Phys. A: Math. Gen. 39 10375-84

[3] Agrawal O P 2007 Fractional variational calculus in terms of Riesz fractional derivatives J. Phys. A: Math. Theor. 40 6287-303

[4] Anthony K H 1986 A new approach to thermodynamics of irreversible processes by means of Lagrangeformalism Disequilibrium and Self-Organisation ed C W Kilmister (Dordrecht: Reidel) pp 75-92

[5] Atanacković T M, Konjik S and Pilipović S 2008 Variational problems with fractional derivatives: EulerLagrange equations J. Phys. A: Math. Theor. 41095201

[6] Atanacković T M, Oparnica Lj and Pilipović S 2007 On a nonlinear distributed order fractional differential equation J. Math. Anal. Appl. 328 590-608

[7] Baleanu D and Avkar T 2004 Lagrangians with linear velocities within Riemann-Liouville fractional derivatives Nuovo Cimento della Societa Italiana di Fisica B 119 73-9

[8] Baleanu D and Trujillo J J 2008 On exact solutions of a class of fractional Euler-Lagrange equations Nonlinear Dyn. 52 331-5

[9] Dacorogna B 2008 Direct Methods in the Calculus of Variations 2nd edn (New York: Springer)

[10] Dreisigmeyer D W and Young P M 2003 Extending Bauer's corollary to fractional derivatives J. Phys. A: Math. Gen. 37 117-21

[11] Enelund M, Mähler L, Runesson K and Lennart Josefson B 1999 Formulation and integration of the standard linear viscoelastic solid with fractional order rate laws Int. J. Solids Struct. 36 2417-42

[12] Filippov V M 1989 Variational Principles for Nonpotential Operators (Translations of Mathematical Monographs vol 77) (Providence RI: American Mathematical Society)

[13] Frederico G S F and Torres D F M 2008 Fractional optimal control in the sense of Caputo and the fractional Nöether's theorem Int. Math. Forum 3 479-93

[14] Gorenflo R, Mainardi F and Vivoli A 2007 Continuous-time random walk and parametric subordination in fractional diffusion Chaos Solitons Fractals 34 87-103

[15] Henneaux M and Teitelboim C 1992 Quantization of Gauge Systems (Princeton, NJ: Princeton University Press)

[16] Jumarie G 2007 Lagrangian mechanics of fractional order, Hamilton-Jacobi fractional PDE and Taylor's series of nondifferential functions Chaos Solitons Fractals 32 969-87

[17] Kilbas A A, Srivastava H M and Trujillo J J 2006 Theory and Applications of Fractional Differential Equations (Amsterdam: Elsevier)

[18] Kwok Sau Fa 2005 A falling body problem through the air in view of the fractional derivative approach Physica A 350 199-206

[19] Miller K S and Ross B 1993 An Introduction to the Fractional Integrals and Derivatives-Theory and Applications (New York: Wiley)

[20] Muslih S I and Baleanu D 2005 Hamiltonian formulation of systems with linear velocities within RiemannLiouville fractional derivatives J. Math. Anal. Appl. 304 599-606

[21] Nakhushev A M 2003 Fractional Calculus and its Applications (Moscow: Fizmatlit) (in Russian)

[22] Oldham K B and Spanier J 1974 The Fractional Calculus (New York: Academic)

[23] Podlubny I 1999 Fractional Differential Equations (Mathematics in Science and Engineering vol 198) (San Diego: Academic)

[24] Sh Rekhviashvili S 2004 The Lagrange formalism with fractional derivatives in problems of mechanics Tech. Phys. Lett. 30 33-7

[25] Riewe F 1996 Nonconservative Lagrangian and Hamiltonian mechanics Phys. Rev. E 53 1890-1899

[26] Riewe F 1997 Mechanics with fractional derivatives Phys. Rev. E 55 3581-3592

[27] Samko S G, Kilbas A A and Marichev O I 1993 Fractional Integrals and Derivatives-Theory and Applications (Amsterdam: Gordon and Breach)

[28] Tarasov V E and Zaslavsky G M 2006 Dynamics of low-level fractionality Physica A 368 399-415

[29] Tofighi A and Golestani A 2008 A perturbative study of fractional relaxation phenomena Physica A 368 1807-17

[30] Vujanović B D and Atanacković T M 2004 An Introduction to Modern Variational Technics in Mechanics and Engineering (Boston, MA: Birkhäuser)

[31] West B J, Bologna M and Grigolini P 2003 Physics of Fractal Operators (New York: Springer) 